\title{
高阶相互作用对宿主-寄生群落动态的影响
}

\author{
高志灵, 苏 敏 ${ }^{*}$, 江正俊 \\ 合肥工业大学数学学院, 合肥 230601
}

\begin{abstract}
摘要:物种间相互作用是影响生物群落稳定性和多样性的重要因素。基于 Lotka-Volterra 竞争模型,通过构建多宿主种群的种 内和种间高阶相互作用模型,研究宿主种群的间接竞争效应对寄生群落动态的影响机制。为有效地揭示高阶作用对种群动态 的影响, 通过对比宿主-寄生群落的现象模型以及机制模型,利用机制模型产生的合理数据集对现象模型中高阶项的参数进行 拟合, 进而探讨了高阶相互作用在群落动态中的作用。结果显示, 完整的高阶相互作用模型在描述多宿主-寄生系统的群落动 态中表现最优, 而直接相互作用模型对群落动态的描述相对较差, 即同时考虑种间和种内的高阶相互作用模型更加符合机制模 型所描述的群落动态。此外,种内高阶作用和种间高阶作用产生不对称效应,宿主间的种间高阶作用对群落产生的影响较种内 高阶作用更为显著。该研究结果在一定意义上丰富了宿主-寄生生物群落的稳定性研究,为理解物种间相互作用的多样性研究 提供了依据。
\end{abstract}

关键词: 高阶相互作用;间接效应;宿主-寄生系统;机制模型;现象模型

\section{Effect of higher-order interactions on the community dynamics of host-parasite system}

GAO Zhiling, SU Min* , JIANG Zhengjun

School of Mathematics, Hefei University of Technology, Hefei 230601, China

\begin{abstract}
Broad evidence has shown that species interactions could influence the stability and diversity of ecological communities. Higher-order interactions ( HOIs) were defined as non-additive effects of density on per captia growth of species. Previous studies have showed that the non-additive effects could strongly influence community stability, patterns of diversity, and better explaining the dynamics of natural community. However, the effect of higher-order interactions on the parasitic community has received little attention. Following this line of thinking, we examined the role of higher-order interactions in the dynamics of multihost-parasite community. We first extended the Lotka-Volterra competition model into the phenomenological models describing a multihost community with three host species that incorporated a generalist, environmentally transmitted parasite. To explore the implications of higher-order interactions for the parasitic infection, we then included the intra-specific and/or inter-specific higher-order interactions in the multihost-parasite system. Phenomenological models can tell us something about where, when, and what of higher-order interactions, but they tell us little about how and why. We supposed these interactions arising via resource competition. Then, we further constructed the mechanistic models of resource competition of multihost-parasitic community, in which non-additive dynamics emerged implicitly, without the need to invoke the higher-order terms. Data generated from simulations of mechanistic models to assess the frequency and magnitude of higher-order interactions arising about the dynamics of multi-host system. Then, we fitted the four phenomenological models by regression analysis: (i) direct interactions model; (ii) intra-specific higherorder interactions model; ( iii ) inter-specific higher-order interactions model; and (iv) fully specified higher-order
\end{abstract}

基金项目:国家自然科学基金项目(31770470)

收稿日期:2020-04-12; 网络出版日期:2021-01-27

*通讯作者 Corresponding author.E-mail: sum04@163.com 
interactions model (including intra-specific and inter-specific higher-order interactions). The results revealed that the fit goodness displayed different results with models of higher-order interactions and direct interactions. Importantly, fully specified higher-order interactions model performed best in describing the community dynamic of multiple host-parasitic, but the direct interactions model showed the worst result among the four models. In addition, the results also revealed that the effects of intra-specific and inter-specific higher-order interactions were asymmetric, and the model with inter-specific higher-order interactions predicted accurately than that of intra-specific higher-order interactions. In order to test the sensitivity and robustness of the dataset generation and fitting process, we conducted additional simulations by changing perturbation densities to less than half of each host's carrying capacity. And the results showed that the simulation method is reasonable. Our results thus underlined the importance of considering higher-order interactions when investigating the multihost-parasite community, and provided a basis for understanding the diversity of interactions between species.

Key Words : higher-order interactions; indirect interactions; host-parasitic system; mechanistic model ; phenomenological model

物种间相互作用是影响生物群落多样性模式的重要因素 ${ }^{[1-4]}$ 。已有的理论研究工作大多是基于物种间 的直接相互作用模型来探究物种的共存和多样性 ${ }^{[5-9]}$, 这些模型虽然应用广泛, 但是模型结果与实验数据的 吻合还不够准确 ${ }^{[10-15]}$ 。近年来, 物种间的高阶相互作用 (higher-order interactions, 缩写 HOIs) 逐渐引起生态学 家的关注 ${ }^{[12,16-18]}$ 。高阶相互作用是指相互竞争的两物种之间的相互作用对第三个物种的增长率所起到的非 累加效应 ${ }^{[12,18]}$ 。高阶相互作用在生物群落中的重要性一直是群落生态学所关注的焦点问题之一, 实验方面 的研究工作亦对高阶相互作用的存在进行详细的描述 ${ }^{[12,16,19-22]}$ 。理论生态学方面, 高阶相互作用在种群竞争 模型中的重要性也不断得到发展 ${ }^{[12,18]}$ 。Bairey 等 ${ }^{[17]}$ 采用含高阶相互作用的复制动态模型, 论证了在随机相 互作用的群落中高阶相互作用对群落多样性和稳定性的影响与只考虑直接相互作用时的结果相反。Grilli 等 ${ }^{[23]}$ 和 AlAdwani 和 Saavedra ${ }^{[24]}$ 证明了高阶相互作用能够增加群落的多样性和稳定性。Mayfield \& Stouffer ${ }^{[12]}$ 结合实验数据, 利用负二项式模型, 证明了含高阶相互作用的植物群落模型相对其他模型更加符合 实验数据。对于寄生群落,宿主间同样存在竞争资源的现象,这种高阶相互作用如何影响寄生群落的动态?

本文在已有的研究基础上 ${ }^{[18]}$, 构建了多物种宿主寄生群落模型,假设宿主间通过共享同一种资源而存在 相互竞争关系, 宿主在消耗资源时, 可能会受到环境中的寄生生物 (free-living parasite) 的感染 ${ }^{[25-28]}$, 从而增加 了宿主的死亡率。当已感染宿主死亡后, 就会将寄生生物释放到其体外环境中, 再次成为自由生活阶段的寄 生生物 ${ }^{[25-26]}$ 。基于易感宿主、已感染宿主及环境中寄生生物动态的理论模型, 探讨高阶相互作用在多物种宿 主-寄生群落中的重要性。

\section{1 模型构建}

首先, 基于 Lotka-Volterra 竞争模型 ${ }^{[12-15,18,24]}$, 本文构建了存在资源竞争关系的多物种宿主寄生系统,为了 研究高阶相互作用的影响, 分别考虑了如下四种现象模型 ${ }^{[18]}:$ (I) 直接相互作用模型 (direct interactions model), 即只考虑直接成对的物种间竞争作用 (图 1); (II) 种内 HOIs 模型 (intra-specific HOIs model), 即考虑 宿主种内相互作用对另一物种的累积效应对其增长引起的间接作用 (图 1); ( III) 种间 HOIs 模型 (interspecific HOIs model), 即考虑两物种间相互作用对第三个物种的累积效应对其增长所起到的间接作用; ( IV) 完整的 HOIs 模型 (fully specified HOIs model), 即同时考虑种内和种间的高阶作用模型。为了检验上述四种 模型中哪一个可以更好地描述多宿主寄生群落, 本文进一步构建涵盖完整资源竞争过程的机制模型, 并分别 对比机制模型和上述四种现象模型,探讨高阶相互作用对寄生生物群落中种群动态的作用。

\section{1 现象模型}

假设宿主间是资源利用性竞争, 同时宿主受到环境中自由生活状态的寄生生物感染威胁, 因此, 根据寄生 生物的传播途径对理论模型做如下假设: (I) 宿主分为易受感染的宿主 $\left(S_{i}, i=1,2,3\right)$ 和已感染发病的宿主 


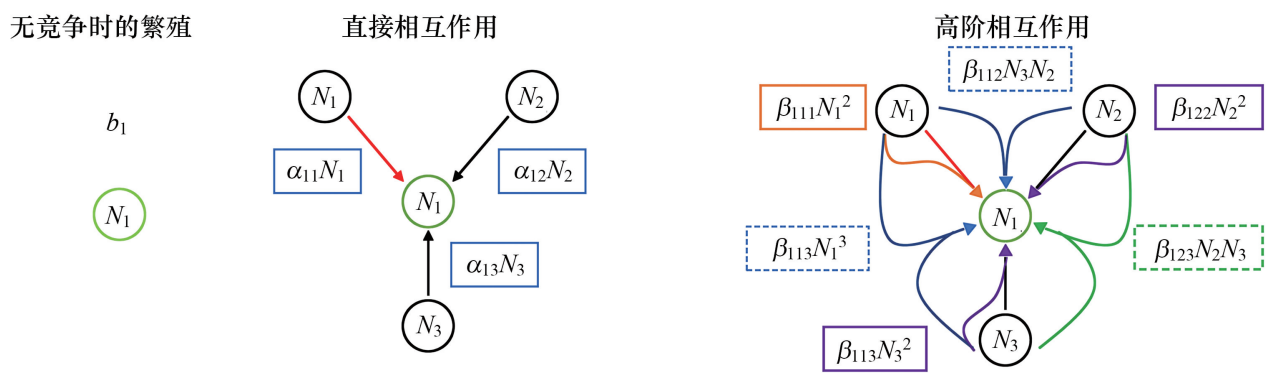

$N_{i}(i=1,2,3)$ 宿主种群; $b_{i}(i=1,2,3)$ 出生率; $\alpha_{1 j} N_{j}(i=1,2,3)$ 直接作有 $; \beta_{1 j k} N_{j} N_{k}(j, k=1,2,3)$ 高阶作用

图 1 宿主物种间的相互作用对个体生殖的影响

Fig.1 Effects of host interactions on the reproduction of population individuals

红色箭头代表宿主 1 个体间的种内相互作用,黑色箭头代表宿主 $i(i=2,3)$ 的个体与宿主 1 的个体间的直接种间相互作用; 橙色和紫色代 表种内高阶相互作用,蓝色和绿色代表种间的高阶相互作用

$\left(I_{i}, i=1,2,3\right)$ 两类; ( II) 宿主的增长是密度依赖的自然增长, 易感宿主和已感染宿主具有相同的繁殖后代的 能力。由于寄生感染不是垂直传播的途径, 因此假设已感染宿主的后代仍然是健康的易感染宿主; ( III) 宿主 间的资源竞争采用 Lotka-Volterra 模型, 易感宿主和已感染宿主均具有竞争能力; ( IV) 易感染宿主因环境中自 由生活的寄生生物 $(E)$ 而发生感染, 寄生感染将会引起已感染宿主发生额外的死亡。当已感染宿主死亡后, 体内的寄生生物被释放到其体外进人环境, 进而转化成自由生活阶段。基于上述假设, 当只考虑宿主间的直 接相互作用时,我们可以用下面的现象模型描述寄生感染系统:

$$
\begin{aligned}
\frac{\mathrm{d} S_{i}}{\mathrm{~d} t} & =b_{i}\left(S_{i}+I_{i}\right)\left[1-\sum_{j=1}^{3} \alpha_{i j}\left(S_{j}+I_{j}\right)\right]-\gamma_{i} S_{i} E-\mu_{i} S_{i} \\
\frac{\mathrm{d} I_{i}}{\mathrm{~d} t} & =\gamma_{i} S_{i} E-\left(\mu_{i}+\nu_{i}\right) I_{i} \\
\frac{\mathrm{d} E}{\mathrm{~d} t} & =\sum_{i=1}^{3} \lambda_{i} I_{i}-\sum_{i=1}^{3} \gamma_{i} S_{i} E-d E
\end{aligned}
$$

式中, $b_{i}(i=1,2,3)$ 是第 $i$ 个宿主的自然出生率, $\alpha_{i j}(i, j=1,2,3)$ 是物种间竞争系数, $\gamma_{i}(i=1,2,3)$ 是环境 中自由生活的寄生生物对第 $i$ 个易感宿主的感染率, $\mu_{i}, v_{i}(i=1,2,3)$ 分别代表第 $i$ 个宿主的自然死亡率以及 已感染宿主因寄生感染导致的额外死亡率, $\lambda_{i}(i=1,2,3)$ 是寄生生物在已感染宿主中的释放率; $d$ 是环境中 寄生生物的自然死亡率。

为了探讨高阶相互作用的重要性, 本文通过扩展上述直接相互作用模型, 加人高阶相互作用项 ${ }^{[12,18]}$ 。当 考虑同种宿主个体间的相互作用对所关注物种的影响时, 扩展模型 (1)得到种内高阶相互作用的现象模型, 影响强度用参数 $\beta_{i j j}$ 来体现; 当考虑不同宿主间的相互作用对所关注物种的影响时, 可得到种间高阶相互作用 模型, 高阶作用的强度用参数 $\beta_{i j k}$ 来表示; 当同时考虑种内和种间高阶相互作用, 扩展模型 (1) 可得到如下完 整的高阶作用现象模型:

$$
\begin{aligned}
\frac{\mathrm{d} S_{i}}{\mathrm{~d} t} & =b_{i}\left(S_{i}+I_{i}\right)\left[1-\sum_{j=1}^{3} \alpha_{i j}\left(S_{j}+I_{j}\right)-\sum_{j=1}^{3} \beta_{i j}\left(S_{j}+I_{j}\right)^{2}-\sum_{j=1}^{3} \sum_{k=j+1}^{3} \beta_{i j k}\left(S_{j}+I_{j}\right)\left(S_{k}+I_{k}\right)\right]-\gamma_{i} S_{i} E-\mu_{i} S_{i} \\
\frac{\mathrm{d} I_{i}}{\mathrm{~d} t} & =\gamma_{i} S_{i} E-\left(\mu_{i}+v_{i}\right) I_{i} \\
\frac{\mathrm{d} E}{\mathrm{~d} t} & =\sum_{i=1}^{3} \lambda_{i} I_{i}-\sum_{i=1}^{3} \gamma_{i} S_{i} E-d E
\end{aligned}
$$

式中, $\beta_{i j j}(i, j=1,2,3)$ 是宿主 $j$ 的种内相互作用对物种 $i$ 的累积效应, $\beta_{i j k}(i, j, k=1,2,3, j \neq k)$ 代表物种 $(j, k)$ 间的相互作用对物种 $i$ 的累积效应,其他参数的意义同模型 $(1)$ 。 


\section{2 机制模型}

为了探索高阶间接作用对种群动态的影响,本文进一步构建资源利用性竞争的机制模型。模型假设宿主 之间共享资源 $(R)$ 实现相互竞争, 而宿主间的资源竞争作用通过消耗资源来反馈对宿主增长率的影响, 因 此,机制模型隐含地考虑了现象模型中宿主间的直接竞争和间接竞争关系,体现了一个完整的生态过程。假 设资源 $(R)$ 服从 logistic 增长,已感染宿主具有对资源的竞争能力, 即易感染宿主和已感染宿主均可消耗资 源。根据上述假设可构造如下机制模型：

$$
\begin{aligned}
& \frac{\mathrm{d} S_{i}}{\mathrm{~d} t}=e_{i} f_{i} R\left(S_{i}+I_{i}\right)-\gamma_{i} S_{i} E-\mu_{i} S_{i} \\
& \frac{\mathrm{d} I_{i}}{\mathrm{~d} t}=\gamma_{i} S_{i} E-\left(\mu_{i}+v_{i}\right) I_{i} \\
& \frac{\mathrm{d} E}{\mathrm{~d} t}=\sum_{i=1}^{3} \lambda_{i} I_{i}-\sum_{i=1}^{3} \gamma_{i} S_{i} E-d E \\
& \frac{\mathrm{d} R}{\mathrm{~d} t}=r R\left(1-\frac{R}{K}\right)-\sum_{i=1}^{3} f_{i} R\left(S_{i}+I_{i}\right)
\end{aligned}
$$

式中, $e_{i}(i=1,2,3)$ 是第 $i$ 个宿主 (包含易感宿主和已感染宿主) 捕食资源 $(R)$ 的转化率, $f_{i}(i=1,2,3)$ 代 表宿主种群 $i$ 对资源的消耗率。为了简便, 不妨设 $f_{i}(i=1,2,3)=f \circ r, K$ 分别代表资源 $(R)$ 的自然增长率 及环境容纳量, 其他参数的意义和现象模型相同。

\section{2 数值模拟与结果分析}

基于 Letten 等 ${ }^{[18]}$ 的方法,利用上述三宿主寄生群落的机制模型产生数据集, 然后对相应的宿主-寄生现 象模型中高阶作用的强度进行参数估计, 通过数值模拟的方法探讨高阶项的引人如何调节现象模型和机制模 型的吻合程度。首先,基于表 1 中的参数对机制模型模拟 $m$ 次 $(m=100)$, 每次模拟均使得所有物种达到稳 定共存, 模拟所用的时间间隔为 $\Delta t=0.1$ 的步长, 记录每步所对应的种群密度, 其中时间 $t \in[0, T]$ 。因参数 $f$ 在每次模拟都是均匀分布下的随机值, 那么对这 $m$ 个 $f$ 的随机参数值来分别计算宿主 $i$ 的承载能力, 即假定 物种 $j$ 的初始时刻密度 $N_{j}(j \neq i)=0$, 模拟机制模型使其达到平衡状态, 记录平衡时刻宿主 $i$ 的种群密度 $N_{i}(T)$, 并记其为宿主 $i$ 的承载能力 $C_{i}(i=1,2,3)$ 。然后, 将承载能力区间 $\left[1, \min \left\{C_{i}(i=1,2,3)\right\}\right]$ 均匀 分为 5 份, 同理, 对承载能力区间 $\left(\min \left\{C_{i}(i=1,2,3)\right\}, \max \left\{C_{i}(i=1,2,3)\right\}\right]$ 再均匀分为 5 份, 得到包含 10 个元素的承载能力序列。

表 1 模型中各参数意义及其取值

Table 1 Definition of the parameters used in the models

\begin{tabular}{lll}
\hline $\begin{array}{l}\text { 参数 } \\
\text { Parameter }\end{array}$ & $\begin{array}{l}\text { 定义 } \\
\text { Definition }\end{array}$ & $\begin{array}{l}\text { 参数范围 } \\
\text { Default value }\end{array}$ \\
\hline$e_{i}(i=1,2,3)$ & 宿主捕食资源的转化率 & $e_{1}=0.1142, e_{1}=0.127, e_{3}=0.14$ \\
$f_{i}=f(i=1,2,3)$ & 宿主对资源的消耗率 & $f \sim$ unifrnd $(0.07,0.145)$ \\
$\gamma_{i}(i=1,2,3)$ & 寄生对易感染宿主的感染率 & $\gamma_{1}=0.505, \gamma_{2}=0.6, \gamma_{3}=0.7$ \\
$\mu_{i}(i=1,2,3)$ & 宿主的自然死亡率 & $\mu_{i}(i=1,2,3)=0.5$ \\
$v_{i}(i=1,2,3)$ & 寄生感染引起的额外死亡率 & $v_{1}=0.1, v_{2}=0.2, v_{3}=0.3$ \\
$\lambda_{i}(i=1,2,3)$ & 已感染宿主的病毒释放率 & $\lambda_{1}=0.5, \lambda_{2}=0.6, \lambda_{3}=0.9$ \\
$r$ & 资源的自然增长率 & 4 \\
$K$ & 资源的环境容纳量 & 100 \\
$d$ & 环境中寄生的自然死亡率 & 0.1 \\
\hline
\end{tabular}


最后, 对机制模型按照如下步骤进行扰动: (I) 各宿主(包含易感染和已感染宿主)在上述承载能力的序 列中随机取值, 得到扰动的全排列, 共有 $A_{10}^{3}$ 组的宿主种群密度组合。其中, 根据实际意义, 排除宿主的密度 取值大于其承载能力的组合; (II) 在上述参数下, 运行机制模型到 $T$ 步时 (多次模拟显示, 种群在 $T=1000$ 达 到稳定, 即进行 10000 次数值迭代), 按照第 I 步中的种群密度同时对易感染和已感染宿主的密度进行扰动, 每组随机参数下,扰动次数等于物种可取的排列个数; ( III) 扰动后继续运行 $T$ 时间步, 群落重新达到稳定。 因此,基于该模拟过程我们可得到随机产生的 100 组宿主种群密度的时间序列。基于上述模拟过程,随机选 择宿主对资源的消耗率 $f$ 的一个取值, 对机制模型进行一次扰动下的种群动态如图 2 所示。

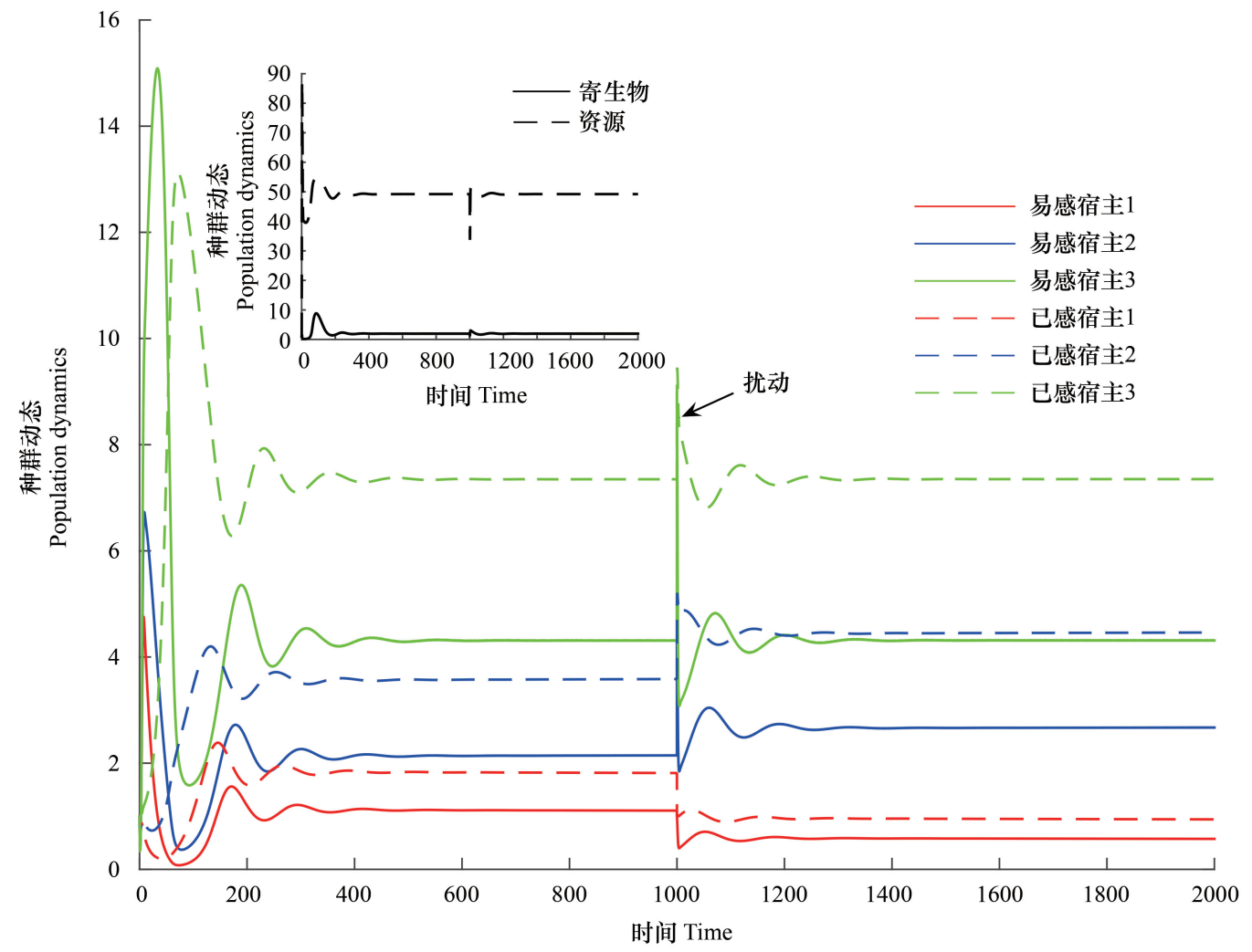

图 2 机制模型下的种群在扰动前后的时间动态

Fig.2 Population dynamics under perturbation with the mechanistic model

参数取值: $e_{1}=0.1142, e_{1}=0.127, e_{3}=0.14, f \sim$ unifrnd $(0.07,0.145), \gamma_{1}=0.505, \gamma_{2}=0.6, \gamma_{3}=0.7, \mu_{i}(i=1,2,3)=0.5, v_{1}=0.1, v_{2}$ $=0.2, v_{3}=0.3, \lambda_{1}=0.5, \lambda_{2}=0.6, \lambda_{3}=0.9, r=4, K=100, d=0.1$

基于上述过程可得到宿主在 [1000,2000] 时间内的密度序列，即 $N_{i}(T+k \Delta t), i=1,2,3$, 选取该时间序 列作为现象模型的样本数据, 记作训练集 (training dataset)。为了减少误差, 本文模拟过程中保持机制模型中 宿主的自然死亡率和疾病致死率与现象模型中的一致。然后,基于该密度的时间序列利用样条差值模型的一 阶导数来计算各宿主密度的变化率, 即 $\frac{d N_{i}}{d t}(i=1,2,3)$ 。最后, 根据所求得宿主种群密度的变化率, 利用多 元回归分析对四种现象模型中的参数进行估计。

同时, 为了检验训练集的样本数据拟合出来的参数对机制模型产生的其他数据是否吻合, 我们构造了新 的密度时间序列作为测试集 (testing dataset)。构造方法如下: (I) 选择 $T=1000$ 时种群在平衡状态的密度作 为机制模型方程的初值; (II) 运行机制模型并记录模拟 50 时间段中间隔 $\Delta t=0.001$ 的时间步长所对应的种群 密度,记录此密度时间序列作为测试集。根据训练集估计的现象模型参数,在相同的初值条件下对现象模型 
进行数值模拟, 分别记录对应的训练集和测试集每时间步上的宿主种群的密度,采用拟合优度的检验方法, 来 检验高阶相互作用模型是否能更精确的描述宿主-寄生群落的动态。这里,我们采用计算多重可决系数 $R^{2}$ 来 衡量拟合优度,具体计算公式如下:

$$
R^{2}=1-\frac{\sum\left(Y_{i}-Y^{\prime}{ }_{i}\right)}{\sum\left(Y_{i}-\bar{Y}_{i}\right)^{2}}
$$

式中, $Y_{i}$ 是机制模型中宿主的密度时间序列; $Y^{\prime}{ }_{i}$ 是现象模型中宿主的密度时间序列; $\bar{Y}_{i}$ 是机制模型宿主密度 的均值。当 $R^{2}$ 越接近 1 , 则拟合效果越好, 即选择的现象模型和机制模型的种群动态吻合的更好。为了更加 直观地看出 HOIs 模型与直接相互作用模型的拟合差距, 本文同时计算了精度差, 即 $\Delta R^{2}=R_{\mathrm{HOIs}}^{2}-R_{\mathrm{Direct}}^{2} \circ \Delta R^{2}$ 为正值, 说明 HOIs 模型比直接相互作用的现象模型对机制模型具有更高的相似度; 反之, $\Delta R^{2}$ 为负值, 直接相 互作用的现象模型与机制模型吻合度更高。

模拟结果揭示, 同时考虑种内和种间 HOIs 模型在描述多宿主-寄生系统的群落动态中表现最优 (图 3)。 训练集和测试集的精度差模拟结果均表明,完整的 HOIs 模型所对应的 $R^{2}$ 与直接相互作用模型的 $R^{2}$ 差距最 大, 即该模型与机制模型吻合得最好 (图 3)。含 HOIs 的 3 个模型描述的群落动态相似度较高, 但与直接相互 作用模型所描述的群落动态差距较大。同时, 对比种内及种间 HOIs 现象模型发现, 种间 HOIs 模型相对种内 HOIs 模型的拟合优度更高, 也说明了种间模型对群落动态的影响更为突出。这一特点在测试集中显现得更 加明显 (图 3)。为了说明数据集的产生和拟合的过程具有稳健性(Robustness), 本文进行了半扰动分析 (图 3 ), 即将物种的承载能力变为原来的一半,重复上述模拟过程,所得结果与图 3 吻合,说明我们的结果不依赖 于扰动的选择。
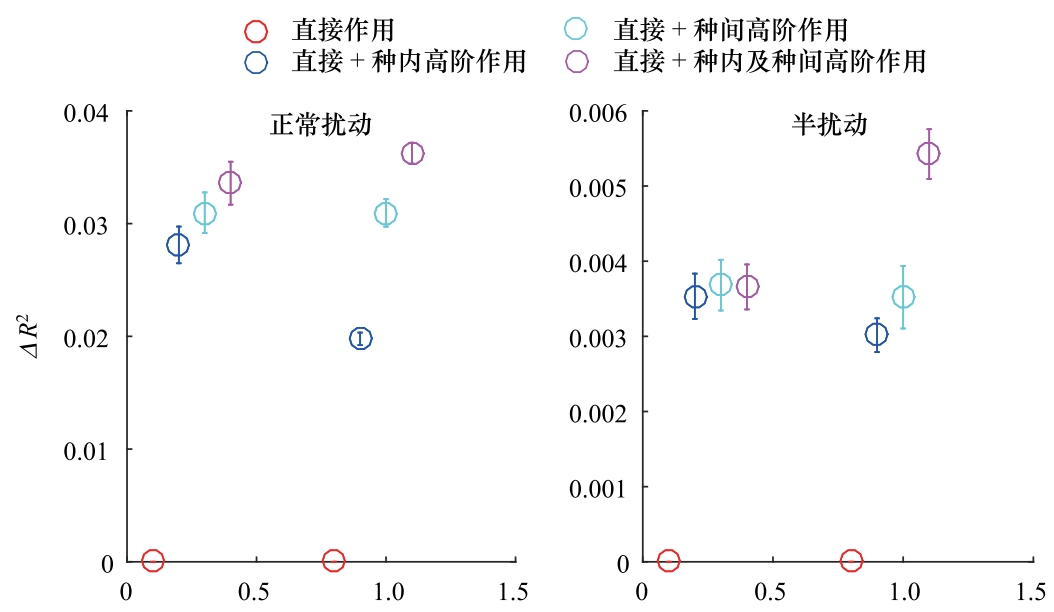

图 3 高阶作用模型与直接相互作用模型的拟合优度差 $\left(\Delta R^{2}\right)$

Fig.3 Difference in goodness of fit between higher-order interactions model and direct interactions model

图中左半部分是由训练数据得到的现象模型与直接相互作用模型的拟合优度差, 右半部分是由测试集数据得到的拟合优度差。参数取值 同图 2

为了更加直观地观察 100 组数据集中, 每一组模型精度的分布情况, 图 4 给出了 HOIs 模型与直接相互作 用模型 $R^{2}$ 的对比连线情况。从图 $4 \mathrm{a}-\mathrm{c}$ 中可以看出, HOIs 模型在描述多宿主-寄生系统的群落动态中普遍表 现更优。100 组数据集中, 每一组都显示 HOIs 模型的 $R^{2}$ 更加接近于 1 , 从而阐明含 HOIs 的现象模型所描述 的群落动态与机制模型的动态更加匹配。对比图 $4 \mathrm{~b}$ 与图 $4 \mathrm{c}$, 发现种间 HOIs 模型的 $R^{2}$ 比种内 HOIs 模型的 $R^{2}$ 集中在更加接近 1 的区域, 说明种间的 HOIs 对群落的动态的影响更为明显, 与图 3 的结果相一致。图 $4 \mathrm{~d}-$ $\mathrm{f}$ 揭示半扰动分析的情况下, 高阶相互作用模型的 $R^{2}$ 更大。虽然半扰动下产生的 100 组数据集中, HOIs 模型 的 $R^{2}$ 出现了比直接相互作用模型 $R^{2}$ 小的情况, 但所占比例非常低 (图 $4 \mathrm{~d}-\mathrm{f}$ 中的红色线)。因此, 结果显示数 
据集的产生和参数估计的过程对本文结果影响不大,也说明物种间的高阶作用能够更好的描述群落动态的结 果具有稳健性。
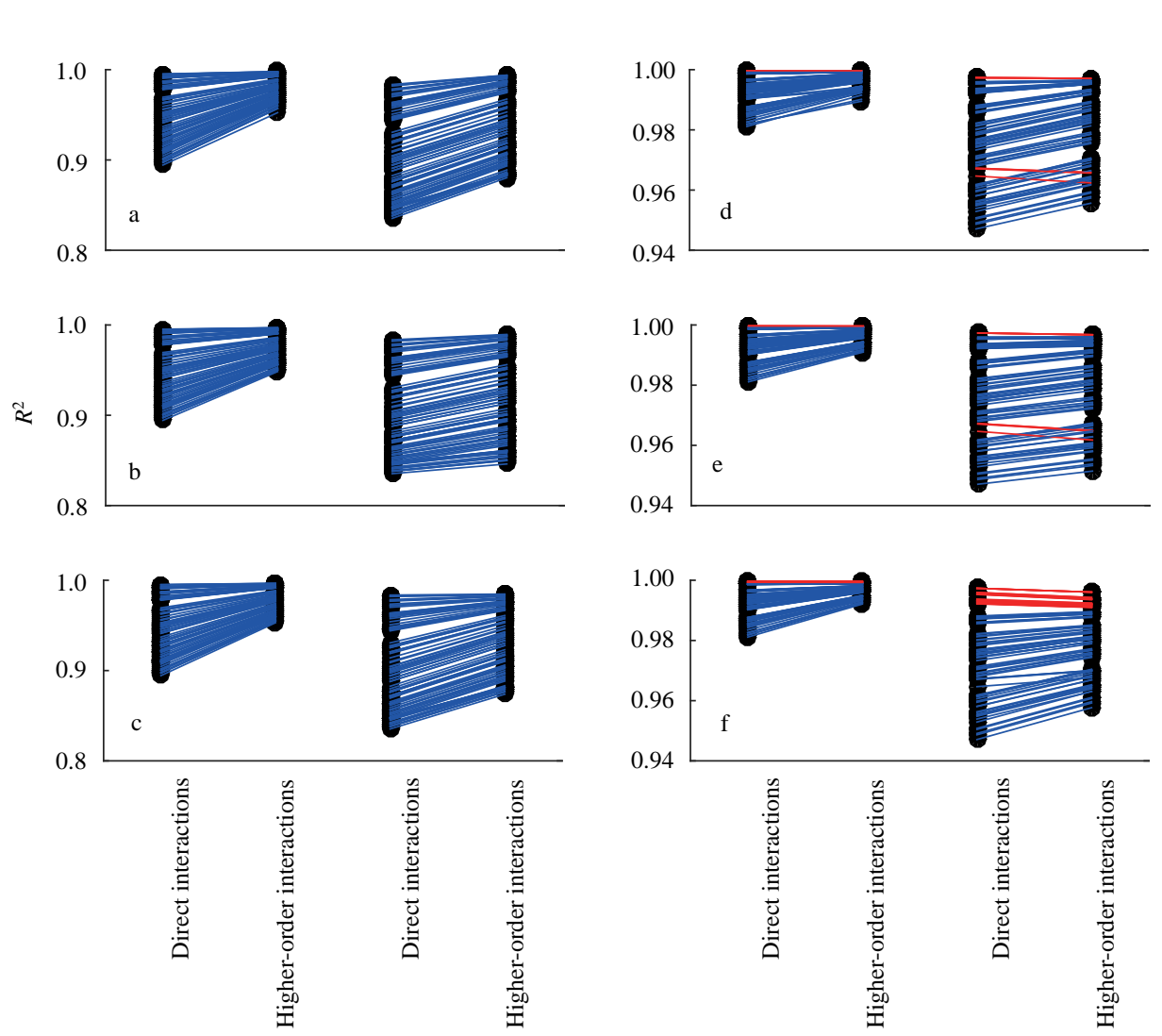

图 4 高阶作用模型与直接相互作用模型 $R^{2}$ 的比较情况

Fig.4 Comparison of the fit goodness between higher-order interactions model and direct interactions model $(\mathrm{a}-\mathrm{c})$ 正常扰动情况下,完整的高阶模型、种内高阶模型以及种间高阶模型与直接作用模型 $R^{2}$ 的比较情况; $(\mathrm{d}-\mathrm{f})$ 半扰动情况下, 完整的高 阶模型、种内高阶模型以及种间高阶模型与直接作用模型 $R^{2}$ 的比较情况。蓝线代表高阶模型的 $R^{2}$ 优于直接相互作用模型的 $R^{2}$, 红线代表 直接作用模型优于高阶模型的 $R^{2}$, 每个子图中左列为训练集产生的结果, 右列为测试集的结果。参数取值同图 2

在前面的分析中,本文在较短时间内评估了不同现象模型的精度,但是在长期状态下,是否会导致现象模 型与机制模型的动态吻合程度较差? 为了评估长期状态下完整的 HOIs 模型与直接相互作用模型的精度, 在 对机制模型进行模拟时将运行时间延长为 4000 ,对参数 $f$ 随机取值 100 次,每次记录时间间隔 $\Delta t=0.1$ 的各宿 主密度的时间序列, 然后根据上述拟合过程, 来拟合完整的 HOIs 模型与直接相互作用模型, 截取最后 1500 步 的种群密度序列进行精度的计算。如图 5 所示, 完整的 HOIs 模型在最后时间段的精度始终更高。因此,结果 揭示完整的 HOIs 模型在长期状态下仍然比直接相互作用模型更加符合机制模型, 进而阐明 HOIs 在多宿主 寄生群落中的影响是不可忽略的。

\section{3 讨论}

群落中间接作用的研究一直备受关注 ${ }^{[10-12,29-30]}$, 然而在竞争群落模型中通常考虑物种间的直接竞争作 用,却忽略了高阶相互作用引起的间接效应对群落动态的影响 ${ }^{[1-33]}$ 。本文在已有的研究方法上 ${ }^{[18]}$ 扩展并构 造了三物种宿主寄生群落, 巧妙地利用宿主-寄生群落的机制模型产生的数据集来确定宿主间高阶相互作用 对群落动态的影响。通过对比机制模型与四种不同类型的现象模型, 研究了宿主间的高阶作用对宿主-寄生 群落动态的影响, 结果揭示在多宿主寄生系统中, 高阶相互作用起到重要作用, 高阶相互作用模型在描述多宿 
主-寄生系统的群落动态中表现较直接相互作用更优。 另外,种内高阶作用和种间高阶作用对拟合优度产生不 对称效应, 宿主间的种间高阶作用对群落产生的影响较 宿主的种内高阶作用更为显著。

本文通过扩展已有的研究方法构造资源利用性竞 争的宿主-寄生机制模型产生样本数据 ${ }^{[12,18]}$, 而不是基 于实验数据的对比, 虽然缺乏实验数据支撑, 但却有利 于减少实验数据测量中一些不可避免的误差。机制模 型通过宿主间对资源的竞争展现了物种间内在的相互 作用,所以该模型所产生的数据具有代表性,研究结果 也显示高阶相互作用的现象模型在训练集与测试集中 都与机制模型产生的数据更为吻合。

以上研究结果对于理解间接作用对宿主-寄生群落 的稳定性影响研究有重要的启迪作用,结果揭示通过调 控两个物种间的相互作用对第三个物种的影响, 能够控

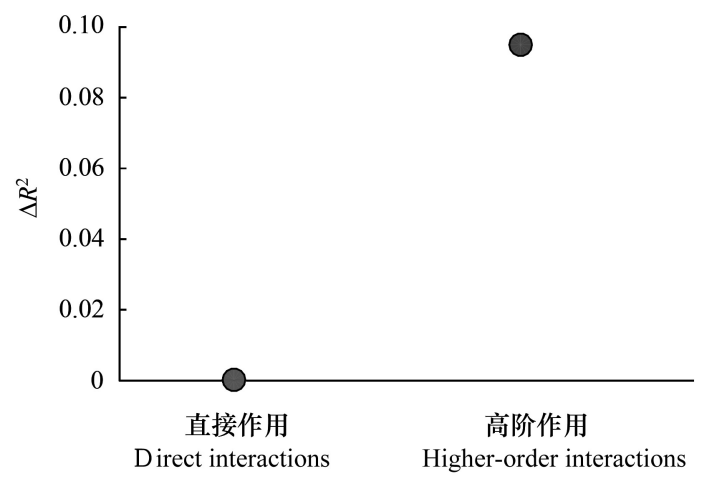

图 5 完整的高阶模型与直接相互作用模型长期状态下的精度 比较

Fig.5 The accuracy of fully specified higher-order interactions model and direct interactions model at long-term behavior 参数取值: $\lambda_{1}=0.5, \lambda_{2}=0.7, \lambda_{3}=0.9$, 其他参数取值与图 2 相同 制宿主种群的数量和寄生生物的多度,从而对寄生的传 播与控制起到重要的作用。然而,本文仅考虑了三宿主与单寄生群落,高阶相互作用对多宿主寄生群落稳定 性的影响待深人研究。

\section{参考文献 (References) :}

[ 1 ] Allesina S, Levine J M. A competitive network theory of species diversity. Proceedings of the National Academy of Sciences of the United States of America, 2011, 108(14): 5638-5642.

[ 2 ] Connolly S R, Roughgarden J. Theory of marine communities: competition, predation, and recruitment-dependent interaction strength. Ecological Monographs, 1999, 69(3): 277-296.

[ 3 ] 姚向阳, 李委涛, 郑玉龙. 群落组成及物种间相互作用对外来植物入侵的影响. 生态学杂志, 2014, 33(7): 1953- 1959.

[ 4 ] Abrams P A. Implications of dynamically variable traits for identifying, classifying, and measuring direct and indirect effects in ecological communities. The American Naturalist, 1995, 146(1) : 112-134.

[ 5 ] HilleRisLambers J, Adler P B, Harpole W S, Levine J M, Mayfield M M. Rethinking community assembly through the lens of coexistence theory. Annual Review of Ecology, Evolution, and Systematics, 2012, 43: 227-248.

[ 6 ] Chesson P. Mechanisms of maintenance of species diversity. Annual Review of Ecology and Systematics, 2000, 31(1) : 343-366.

[ 7 ] Falster D S, FitzJohn R G, Brännström Å, Dieckmann U, Westoby M. Plant: a package for modelling forest trait ecology and evolution. Methods in Ecology and Evolution, 2016, 7(2): 136-146.

[ 8 ] Allesina S, Tang S. The stability-complexity relationship at age 40: a random matrix perspective. Population Ecology, 2015, 57(1): 63-75.

[ 9] 张炜平, 潘莎, 贾昕, 储诚进, 肖酒, 林玥, 白燕远, 王根轩. 植物间正相互作用对种群动态和群落结构的影响: 基于个体模型的研究进 展. 植物生态学报, 2013, 37(6): 571-582.

[10] Wootton J T. The nature and consequences of indirect effects in ecological communities. Annual Review of Ecology and Systematics, 1994, 25( 1) : 443-466.

[11] White E M, Wilson J C, Clarke A R. Biotic indirect effects: a neglected concept in invasion biology. Diversity and Distributions, 2006, 12(4) : 443-455.

[12] Mayfield M M, Stouffer D B. Higher-order interactions capture unexplained complexity in diverse communities. Nature Ecology \& Evolution, 2017, $1(3): 0062$.

[13] Hairston N G, Allan J D, Colwell R K, Futuyma D J, Howell J, Lubin M D, Mathias J, Vandermeer J H. The relationship between species diversity and stability: an experimental approach with protozoa and bacteria. Ecology, 1968, 49(6) : 1091-1101.

[14] Neill W E. The community matrix and interdependence of the competition coefficients. The American Naturalist, 1974, 108(962) : 399-408.

[15] Brenchley G A. Community matrix models: inconsistent results using Vandermeer's data. The American Naturalist, 1979, 113(3): 456-459 
[16] Billick I, Case T J. Higher order interactions in ecological communities: what are they and how can they be detected? Ecology, 1994, 75( 6) : $1529-1543$.

[17] Bairey E, Kelsic E D, Kishony R. High-order species interactions shape ecosystem diversity. Nature Communications, 2016,7 : 12285.

[18] Letten A D, Stouffer D B. The mechanistic basis for higher-order interactions and non-additivity in competitive communities. Ecology Letters, 2019, $22(3): 423-436$.

[19] Vandermeer J H. The competitive structure of communities: an experimental approach with protozoa. Ecology, 1969, 50(3): 362-371.

[20] Levine J M, Bascompte J, Adler P B, Allesina S. Beyond pairwise mechanisms of species coexistence in complex communities. Nature, 2017 , 546 $(7656): 56-64$.

[21] Wootton J T. Putting the pieces together: testing the independence of interactions among organisms. Ecology, $1994,75(6)$ : 1544- 1551.

[22] Case T J, Bender E A. Testing for higher order interactions. The American Naturalist, 1981, 118(6): 920-929.

[23] Grilli J, Barabús G, Michalska-Smith M J, Allesina S. Higher-order interactions stabilize dynamics in competitive network models. Nature, 2017, $548(7666): 210-213$.

[24] AlAdwani M, Saavedra S. Is the addition of higher-order interactions in ecological models increasing the understanding of ecological dynamics? Mathematical Biosciences, 2019, 315: 108222.

[25] Strauss A T, Civitello D J, Cáceres C E, Hall S R. Success, failure and ambiguity of the dilution effect among competitors. Ecology Letters, 2015, $18(9): 916-926$.

[26] Kirk D, Shea D, Start D. Host traits and competitive ability jointly structure disease dynamics and community assembly. Journal of Animal Ecology , $2019,88(9): 1379-1391$.

[27] Al-Shorbaji F, Roche B, Britton R, Andreou D, Gozlan R. Influence of predation on community resilience to disease. Journal of Animal Ecology, $2017,86(5): 1147-1158$.

[28] Rohr J R, Civitello D J, Crumrine P W, Halstead N T, Miller A D, Schotthoefer A M, Stenoien C, Johnson L B, Beasley V R. Predator diversity, intraguild predation, and indirect effects drive parasite transmission. Proceedings of the National Academy of Sciences of the United States of America, 2015, 112(10): 3008-3013.

[29］王红, 苏敏, 潘峰. 寄生对集团内捕食系统中物种人侵的影响. 生态学报, 2016, 36( 15): 4809-4815.

[30] Peacor S D, Werner E E. The contribution of trait-mediated indirect effects to the net effects of a predator. Proceedings of the National Academy of Sciences of the United States of America, 2001, 98( 7) : 3904-3908.

[31] MacArthur R. Species packing and competitive equilibrium for many species. Theoretical Population Biology , 1970 , 1(1): 1-11.

[32] Vandermeer J H. Generalized models of two species interactions: a graphical analysis. Ecology, 1973, 54(4) : 809-818.

[33] Law R, Watkinson A R. Response-surface analysis of two-species competition: an experiment on Phleum arenarium and Vulpia fasciculata. Journal of Ecology, 1987, 75(3): 871-886. 